\title{
Lie, Santilli, and Nanotechnology From the Elementary Particles to the Periodic Table of the Elements
}

Erik Trell

Linköping University Post Print

Tweet

N.B.: When citing this work, cite the original article.

Original Publication:

Erik Trell , Lie, Santilli, and Nanotechnology From the Elementary Particles to the Periodic Table of the Elements, 2014, 10TH INTERNATIONAL CONFERENCE ON MATHEMATICAL PROBLEMS IN ENGINEERING, AEROSPACE AND SCIENCES (ICNPAA 2014), 1100-1109.

http://dx.doi.org/10.1063/1.4904685

Copyright: (C) 2014 AIP Publishing LLC http://scitation.aip.org/

Postprint available at: Linköping University Electronic Press

http://urn.kb.se/resolve?urn=urn:nbn:se:liu:diva-114457 


\section{AIP $\mid$ proceedings}

Lie, Santilli, and nanotechnology: From the elementary particles to the periodic table of the elements

Erik Trell

Citation: AIP Conference Proceedings 1637, 1100 (2014); doi: 10.1063/1.4904685

View online: http://dx.doi.org/10.1063/1.4904685

View Table of Contents: http://scitation.aip.org/content/aip/proceeding/aipcp/1637?ver=pdfcov

Published by the AIP Publishing

\section{Articles you may be interested in}

Foundations of Santilli's Lie-Isotopic Theory

AIP Conf. Proc. 1281, 860 (2010); 10.1063/1.3498623

Viewing the periodic table of the elements with $\mathrm{x}$-rays

Phys. Teach. 39, 235 (2001); 10.1119/1.1367796

Representations of the Weyl Lie algebra as models of elementary particles

J. Math. Phys. 19, 2278 (1978); 10.1063/1.523604

The Elements: An Illustrated History of the Periodic Table

Phys. Today

Two new elements added to the periodic table

Phys. Today 


\title{
Lie, Santilli, and Nanotechnology From the Elementary Particles to the Periodic Table of the Elements
}

\author{
Erik Trell \\ Faculty of Health Sciences, University of Linköping, Se-581 83 Linköping, Sweden. \\ erik.trell@gmail.com
}

\begin{abstract}
Santilli's revolutionary iso-, geno- and hypermathematics have provided the original straight line Lie groups and algebras with a span and coherence in all dimensions, and thus already at the infinitesimal level an extension in the Cartesian sense, allowing a continuous self-similar cyclical realization of matter from the elementary particle threshold level via the atomic to molecular and visible scale where it meets and marries with modern nanotechnology in the form of an isotropic vector matrix of space-filling octahedron-tetrahedron composition. This is distributed as an electron transition matrix with Bohr shell model stratified signature and is here directly outlining a new, centrally coordinated organic composition and chart of the periodic system as specifically exemplified by the noble gases.
\end{abstract}

\section{INTRODUCTION}

In this international year of crystallography, a coinciding important anniversary is that it is 125 years since Wilhelm Killing introduced his Wurzelsystem ${ }^{1}$ in order to classify Marius Lie's then recently disclosed ${ }^{2,3}$ continuous transformation groups and algebras over the field of complex numbers. However, it has more and more emerged that the elegant vector diagrams that the root system comprises directly line up with real Euclidean space, too, where - and in that capacity also referred to as crystallographic - they quite by themselves lay out, combine and perpetuate the manifold lattice arrangement varieties found in Nature already at the infinitesimal level. Attesting this evidence of a coherent nanotechnological aspect and constitution of Nature and giving it canonical universal algorithm, the corresponding effective hybridization into ordinary structural laws and formulas has been realized by the Lie-admissible algebras of Ruggero Maria Santilli's powerful iso-, geno- and hypermathematics. ${ }^{4-6}$ Emulating their principal lifting of the trivial +1 unit into a bi-valued $n$-dimensional isotopic element compound incorporating the factors and operations of the equation into (iso-)unit value, allowed me many years ago in the simplest case of the $\mathrm{A}_{2}$ root vector diagram of $\mathrm{SU}(3)$ to duplicate it with its commutator (Fig. 1) and thus making "according to Santilli...a transition from contemporary physical models based on Lie algebras or their graded-supersymmetric extensions to the general Lie-admissible models, which transition essentially permits the treatment of particles as being extended and therefore admits additional contact, non-potential and non-Hamiltonian interactions. ${ }^{5}$

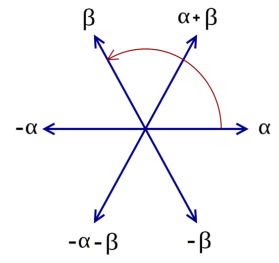

a

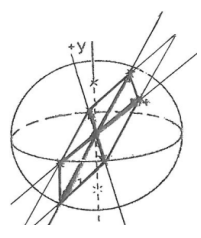

b

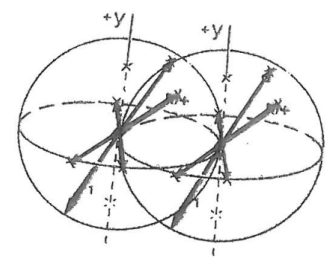

C

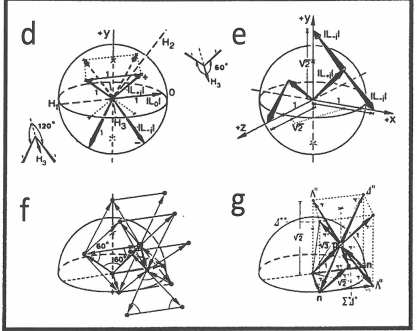

FIGURE 1. When one $A_{2}$ root space diagram (a) is inscribed in the unit sphere the vector network is plane (b), and (c) does not connect three-dimensionally, but when the commutator diagram is accommodated (d) a full Lie-admissible lattice results (e-g). 


\section{METHODS AND RESULTS}

By that effective $\mathrm{SO}(3) \times \mathrm{O}(5)$ automorphic coset decomposition of $\mathrm{SU}(3)$ over the real numbers, a hexagonal spaceframe lattice is generated and octagonally accommodated in classical three-dimensional Euclidean space (Fig. $1 \mathrm{~d}-\mathrm{g}$ ) that is the cubical framework (Fig. 2a) for Lie's original geometriske transformationer "between the Plücker line geometry and a spatial geometry whose element is the sphere" by a "polygon...projection (of the Plücker cubical) space's straight lines into the sphere's rectilinear generatrices" ${ }^{\prime 2,3}$ - which, in turn, are just the duplicated $\mathrm{A}_{2}$ diagram (Fig.2d). ${ }^{7-13}$ As previously reported, this essentially digital 'eightfold eightfold way ${ }^{10-14}$ system, congenial to all provenience - including Higg's ${ }^{13}$ - enables an exhaustive and exact reproduction of the parental 'cuBit' eigenvector field with instant representation and solutions of the Diophantine equations, Fermat's last theorem and Beal's conjecture ${ }^{10,14,15}$ as well as of the elementary particle spectroscopy by volume- and spherical symmetrypreserving infinitesimal "curve-net",2,3 transformations and projections between straight and round therein ${ }^{7-14}$; verbatim as the true Lie prescriptions direct. ${ }^{2,3}$ This has been amply accounted for up to the baryons and mesons but not yet fully in the leptons ${ }^{9-13}$, so the aim of the present paper is to continue with these and in especial the electron and subsequent atomic formations, producing, partially by the principles of octagon-square periodic tiling a new, organic composition and chart of the periodic system as here specifically exemplified by the noble gases.

\section{An Isotropic Vector Matrix Realization of the Electron}

Figure 2 illustrates the outward continuation of the duplicated root vector diagram between the unit sides, in this case the upper left of the eight Cartesian segment 'cuBits' of a Lie algebra neighborhood of Euclidean space (Fig. 2a) "into the sphere's rectilinear generatrices",2,3 (=infinitesimal generators) in one of the eight corners flanking the central spherical Nucleon domain (Fig. 2b,c). Further outward iteration sets up a lattice which in the $60-120^{\circ}$

a

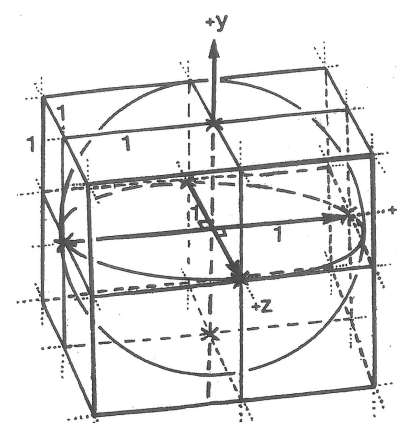

b

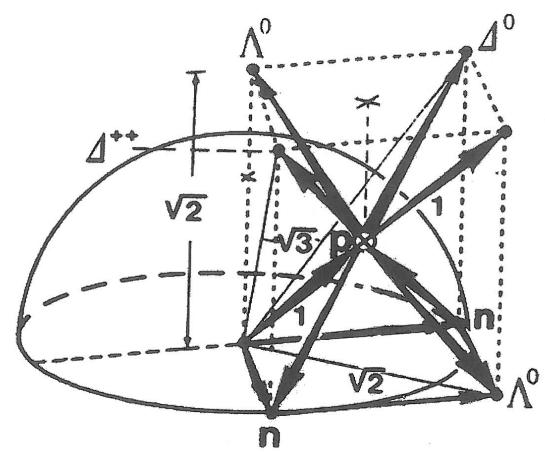

$\mathrm{c}$

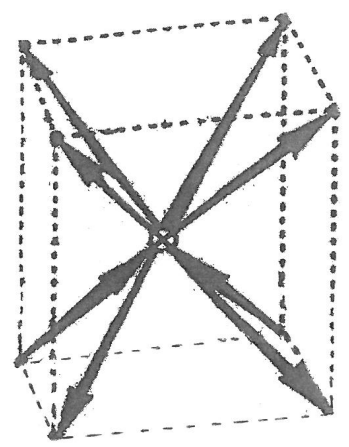

FIGURE 2. a) The generatrix ${ }^{2,3}$ of Euclidean space is the Lie algebra neighborhood of the Plücker straight line geometry outlined by the eight infinitesimal Cartesian segments in it. b) In any of the two times four Cartesian quadrants from and around the spherical nucleon center, here the upper left, the diagonal ("quark-skewed") root vectors span a lattice branching outwards as sections of a space frame isotropic vector matrix (c).

connections next dealt with (figure 3a,b) consists of two half-octahedron sides of charged root vectors alone (figure $2 \mathrm{~b}, \mathrm{c})$. In the case of starting from the upper left Cartesian segment cubit in Fig. 2a one can visualize what happens as that its four vertical sides are deflected $60^{\circ}$ from the sphere's nearest corner vertex, or tangential "image point",3, creating the top of a $2^{1 / 2}$ high octahedron whose sequence continues upwards in a parallelepiped column (Fig. $2 \mathrm{~b}$ ) but may also be horizontally connected, adding two tetrahedrons to an octahedron interval (Fig. 3a) into a combined space-filling 'singlet' coil construction element of twelve unit sides, just as in the cube and like this a regular solid embodiment (Fig. 3b).

However, it is not quite as straightforward as that, because all parent cuBits share all sides with the flanking ones so that in the total transition also the horizontal sides are mobilized in a systematic way, step by step dragging in the neighbors. As shown in the montage on next page (Fig. 3a), when the obtained sections (figure $2 b, c$ ) are threedimensionally tessellated (here just over the $\mathrm{x}$ and $\mathrm{y}$ axes), the resulting lattice is a stacking of the well-known, only other than the cube space-filling unit regular solid constitution, namely, a complex of one octahedron and two tetrahedrons (figure $3 \mathrm{a}, \mathrm{b}$ ). 
a

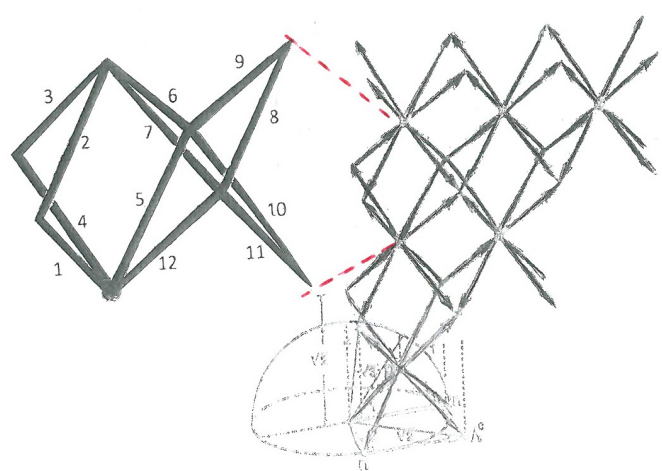

b

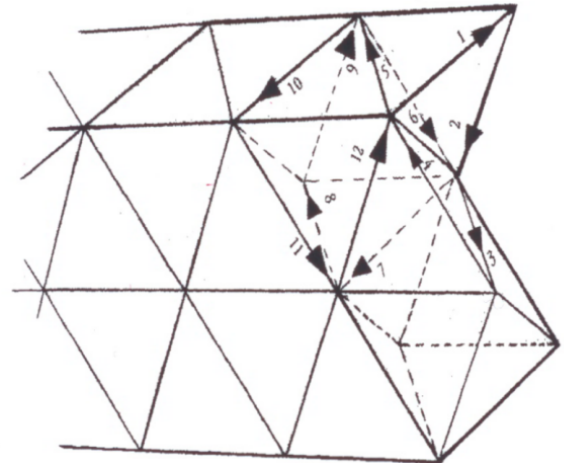

$\mathrm{c}$
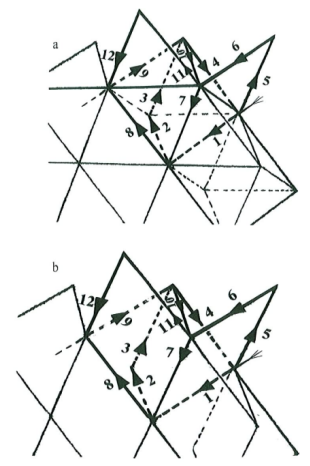

FIGURE 3. (a) A collage of the two-fold half-octagon sections, forming between them an outwards connectable lattice of one-octahedron/two tetrahedrons constitution, which (b) is the only other than the cube space-filling regular solid unit convolution, consisting of equally many, but deflected sides - the horizontal lines are Cartesian coordinate references. In (c), two open-ended varieties of the 12-side "singlet" electron coil are shown, that can continue into the full module.

It consists like the cube of twelve, however, all diagonally 'quark-deflected'10-14 unit sides (the horizontal lines in figure $3 \mathrm{~b}$ are the Cartesian coordinate references). The sideways periodicity, that is, translational symmetry and continuation of the singlet blocks is seen in Fig. 4 a where the next block is the Lie-admissible commutator mirrorimage of the preceding, and thus taking part in forming a both horizontally and vertically periodic lattice. This is an essential nanotechnology structure, whose modular building elements, sections and assembly can thus be realized as a concentrically interlacing phase transition ${ }^{7-13}$ at the very threshold of material arrangement, i.e., an ordinary space frame (Fig. 4b), alias octahedron-tetrahedron or octet truss, whose simultaneous space occupation implies an instant isotropically distributed universal inflation. As seen in figure 3a, the local space frame sections propel from a Cartesian segment of the Lie neighborhood, whose spherical root space thus abides as the hub, which is most pertaining in relation to the octet truss cast because, "viewed mathematically, as a generic pattern, its hubs correspond to the centers of spheres of equal radius". ${ }^{16}$ In uniform lattice distribution this is what Buckminster Fuller called "an isotropic vector matrix (IVM)... with everywhere-the-same energy conditions" and "emphasized over the more familiar xyz apparatus arguing that our fixation on cubes and right angles goes against the grain of nature"(Ib.)

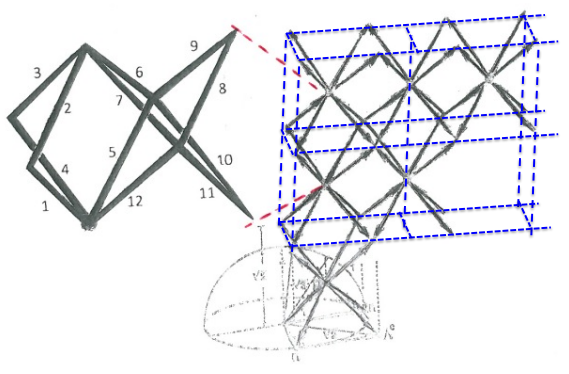

$\mathrm{b}$

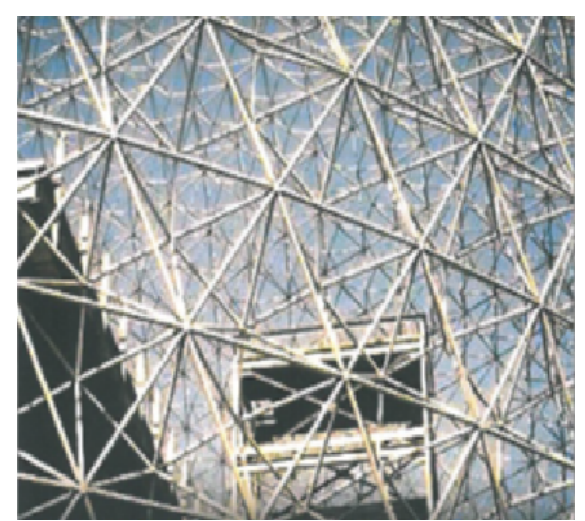

Figure 4. a) Continuation of the root vectors outside the nucleon aligns as a sequence of the space-filling one octahedron/two tetrahedrons regular solid complex with twelve unit sides and translationally as well as vertically periodic and (b) which in iteration goes into a so called octahedron-tetrahedron truss space frame more technically referred to as isotropic vector matrix inclined to hierarchical modular expansion by its central co-ordination.

Once having an isotropic vector matrix, there are many options with important technical, architectural, physical, chemical etc. applications and expansions for space-filling agglomerations, smoothly and without holes fitting in with each other and on the whole. Here, for matters of demonstration, the singlet coils were first mapped into the space-filling truncated octahedron configuration (Fig. 5), giving the exact electron mass relation to the proton, that 


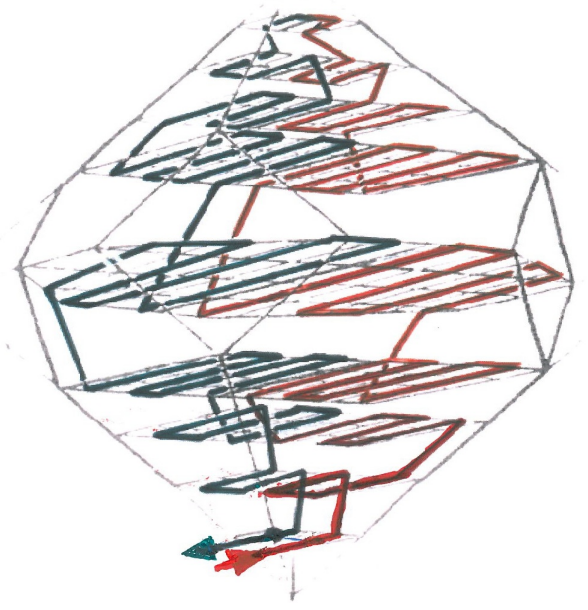

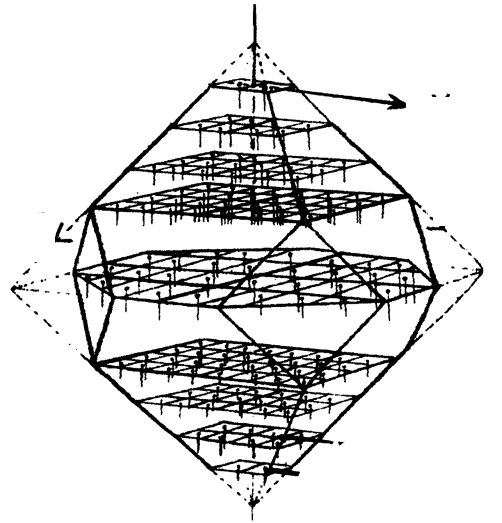

K 2

L $\quad 8$

N 32

$0 \quad 32$

P 32

Q 18

R 8

S 2

152

Mass $=938.28 /(12 \times 152)=0.514 \mathrm{MeV}$

b

FIGURE 5. (a) The two open-ended varieties of the singlet electron coil (Fig. 3c) can join to a non-rotational half-turn continuous delineation of an IVM module, (b) here portrayed as the truncated octahedron, which is a spacefilling Archimedean solid arranged according to palindromic Bohr shell model distribution with the singlet coils in each layer represented as rods.

moves one root vector step while the electron module has made $12 \times 152=1824$ when coming back to the origin for its next cycle.

Figure 6 gives an overview of a direct nanotechnological construction module of the same Bohr periodicity, akin to "the self-organizing specialized bricks" recently described in "robot manipulation of a shared environment... built from square bricks in a non-overlapping grid pattern". ${ }^{17}$

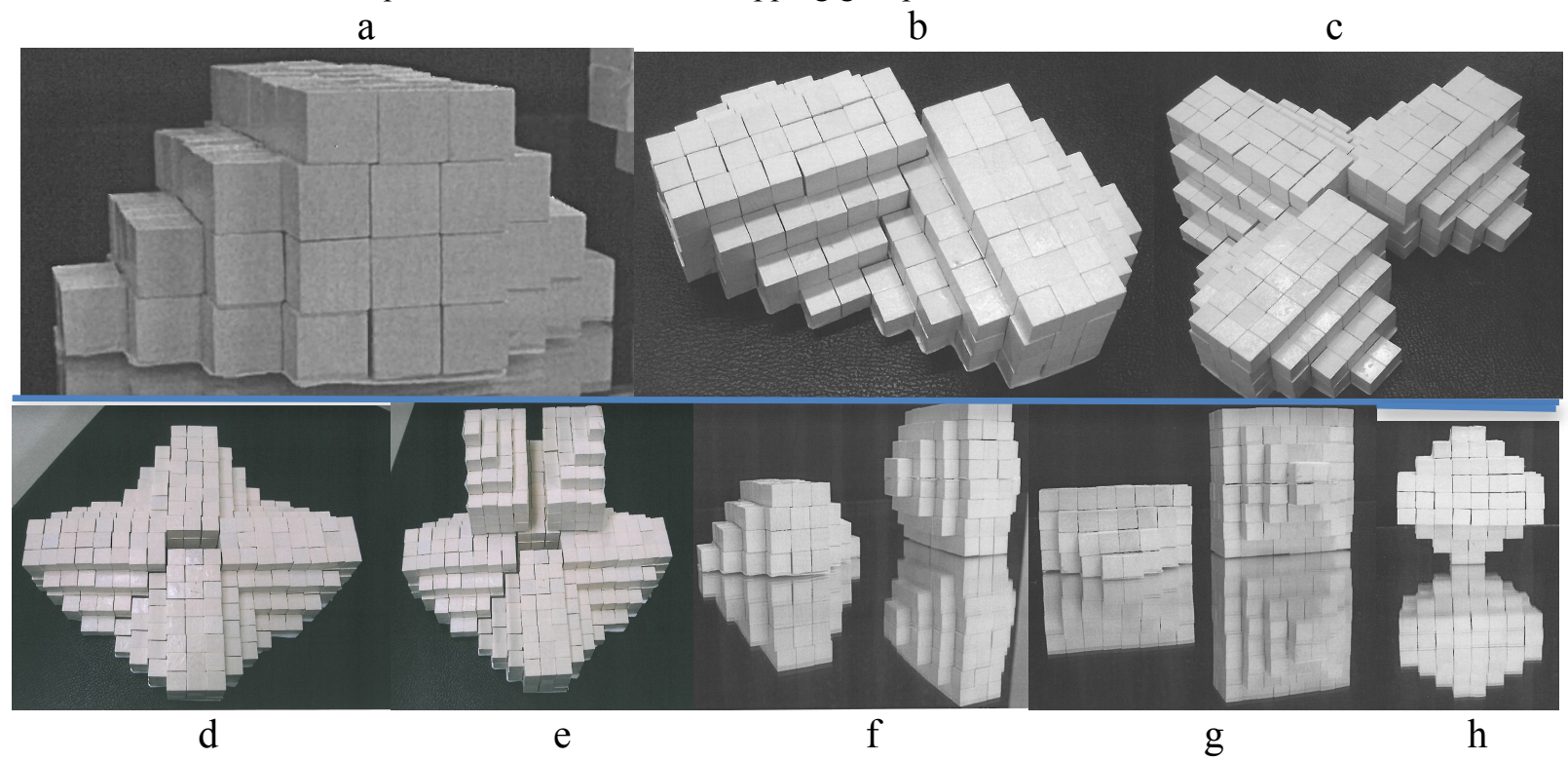

FIGURE 6. (a) The 12-step singlet electron coils are here pieced together as infinitesimal 'cuBits' in a palindromic Bohr orbital model distribution. Actually, they are of parallelepiped span (Fig. 4a) but that doesn't change their continuous linking according to Fig. 5a by a non-rotating Zitterbewegung IVM outline (Fig. 5b) back and forth in apparent time into an altogether 1824-step half-turn module with proportionally higher inertia = lower mass in relation to the central nucleon's unit diagonal = proton and orthogonal $=$ neutron root vectors, and then ready to proceed the modular course (b-e), interchangeably either sideways (b-d), upwards (e) or mirror-wise (f-h). It is seen that the assembly leaves a central vertically (c,d) and horizontally (e) connected groove for the convoying IVM center. In the mirror IVM compounds reciprocal cavities appear (f-g). 
The idea that electrons may be both wave functions, here the cloverleaf singlet coils, and "transition matrix elements" ${ }^{\prime 18}$, here the IVM modules, is not new but in the present cast embodied to a coherent lattice that corresponds to the fact and way that they tangibly weave the world. Like everything else on a digital basis they are but evading unit line bits when pursued to their origin but from there they are caught in the global phase transition "between the Plücker line geometry and a spatial geometry whose element is the sphere" by a "polygon...projection of space's straight lines into the sphere's rectilinear generatrices",2,3 that Lie's original geometriske transformatione in detail define.

The other durable algorithm is the Bohr stratified apportioning of the building blocks whether conceived as points or bodies, and that this pattern proceeds from the elementary particle stage to and beyond the tenthousandfold larger atoms. The mechanism is self-similarity, when the modules both template and fill out a successive cyclical expansion of their motif (Fig. 7a) so that the atom cross-section size is reached within a limited number of cycles.
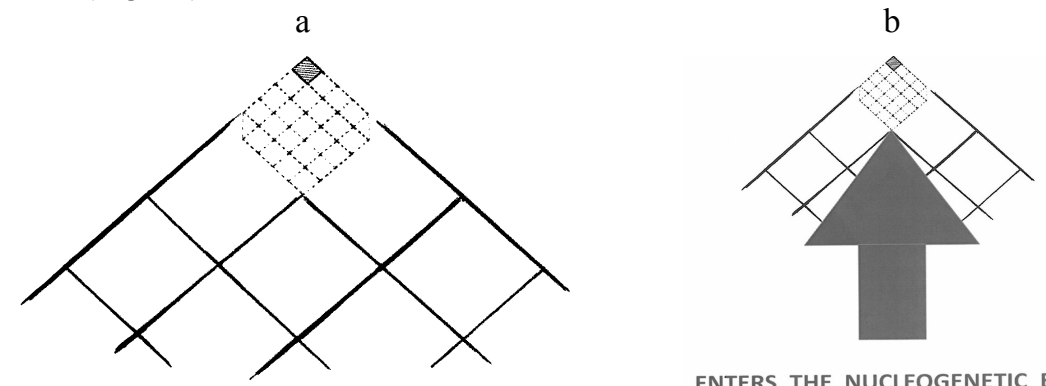

ENTERS THE NUCLEOGENETIC ERA

FIGURE 7. Equatorial plane cross-section of first three cycles of self-templating Hydrogen ion expansion (a) and the dismantling extrusion squeeze exerted by condensation impact in the nucleogenetic era (b).

In the kick-off primordial Universe the serpentine route to the Hydrogen atom that together with some Helium prevails there reminds of the "3D island growth... pathway of layered growth" recently described at "angstrom-scale [and even single-atom scale] resolution" 19,20 in surface crystallization processes, where "the growth units are individual molecules and ions that attach in monomolecular steps present on a crystal surface" (Ib.). That the same modus operandi with identical topographical "step heights, terrace widths, and other surface features" (Ib.) comes up in several cycles from the very ground is then entirely consequential, and the objection that such intricacy cannot happen at such diminutive size can be met by comparing with the myriad times smaller and more complex strings. Quite simply; There is the bottomline unit digit. And it takes so and so many convolutions to shape us ground-up.

But that could not happen in the primordial universe, patches of which still persists, where the island modules peacefully wind up their isotropically dispersed space quota - and nothing more. Inevitably, however, there will be small fluctuations, approaches, collisions, condensations, perturbations, and soon enough the nucleogenetic era ensues, for which there is an explanation, too, in the present model. (Fig 7b) This is that when a number of hydrogen ions are compressed against each other at sufficient energy to fuse, they will be squeezed side by side along a line perpendicular to the force so that from whatever transverse direction will result an axial duplication (Fig. 8a) like in the ends of the mirrored module compounds (Fig. 6 f,g) with Bohr period 2+2, 8+8, 18+18, 32+32 etc.

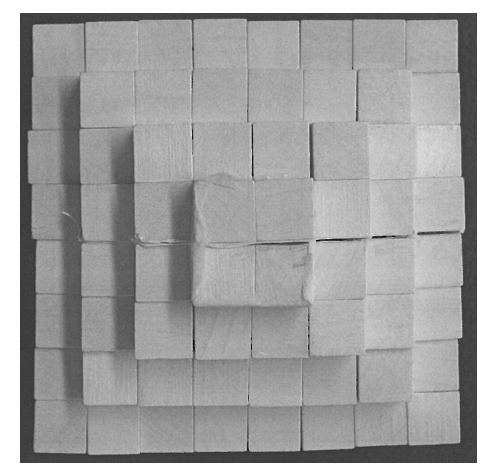

$\mathrm{b}$

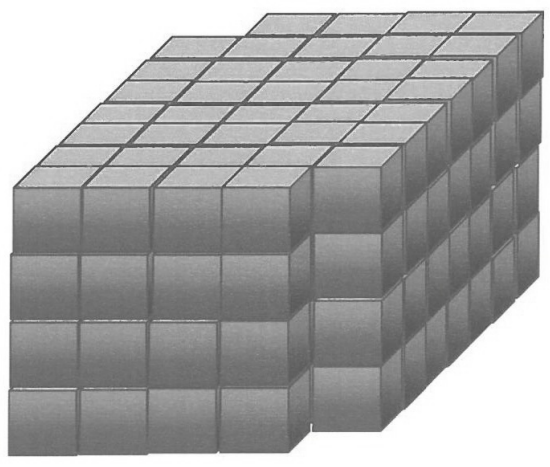

FIGURE 8. a) Mirror juxtaposition of two electron modules with doubled Bohr periodicity. b) By the nucleogenetic pressure the electron module is flattend in its ends, packing the 152 singlets in a square brick with two open corners. 
Due to the pressure the hydrogen ions are forced to uncoil until the last singlet is reached in each, meaning that they come together in the terminal module, where each occupies one singlet in a honeycomb arrangement where the number of cells in duplicated Bohr periodicity depends on how many hydrogen ions were engaged in the fusion.

\section{Periodic Tiling of the Periodic System}

Much of this is of course inferential but we know that there was a primordial Universe of largely Hydrogen, still persisting at places, and we know that there from fully understandably condensation events occurred a still ongoing nucleogenetic era, when the electron IVM fabric underwent the crunch of extreme compression with effects both upon the elements and their arrangement; in which, tentatively, a converging cloud of them is collapsing together in a narrowing module funnel (Fig. 7b) and there cyclically uncoiling under shedding off of photons and neutrinos and other fragments until in the ultimate end pairwise sandwiching up along the sides as a wafer of together equally many singlets as the cloud's amount. Maybe not so crystal-clear formulated - but clear for re-crystallization by the formation of so individualized Hydrogen ion seeds by cross-sectional extrusion in an alternating sequence from the here vertically duplicated Bohr-periodic singlet "terraces" $" 19,20$ (Fig. 8a).

To continue the inferences, when the expansion resumes within these cells, there is still a high pressure causing in the first round a correspondingly contracted electron module (Fig. 8b). This will self-organize and assemble according to the "spiral growth model... of 2D layer growth" (Ib.) that is natural in the composition of the flat, oneor more-storied type of IVM space frame (Fig. 9a) and to which the so modified module is ideally inclined (Fig. 9 $\mathrm{b}, \mathrm{c})$ both in areal and modular expansion by square-octagon periodic tiling ${ }^{21,22}$ and "cube-oct" structure build ${ }^{23}$, respectively; the latter recently reported from the suitably named Center for Atoms and Bits at MIT (Ib.); and both verbatim originally prescribed by Marius Sophus Lie in his thesis "Over en classe geometriske transformationer".,2,3

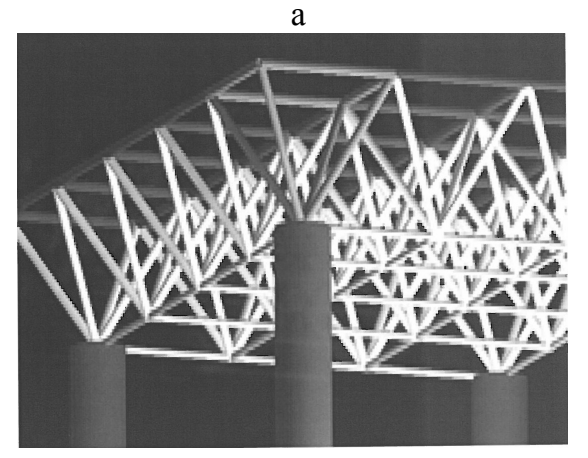

b

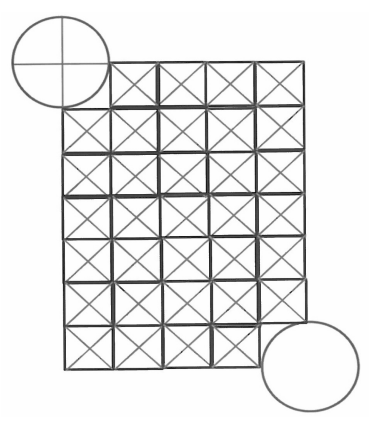

$\mathrm{c}$

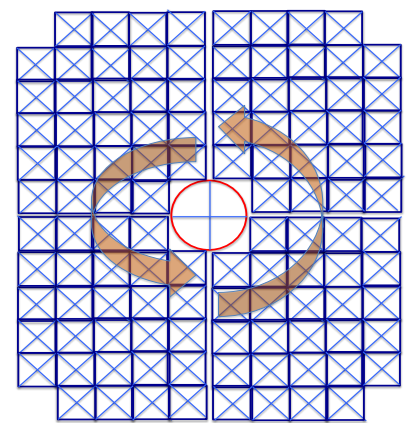

FIGURE 9. a) stratified type of IVM spaceframe (slightly distorted in perspective). b) Cross-section of same type with only two corner beams shown. c) With that self-organized "cube-oct" composition the spiral variety of surface crystal growth can proceed by stacking of the bricks (here not the same number as in the module cross-section) like a flight of stairs in consecutively one layer higher piles, and possible to turn also vertically with then horizontally convoying center.

That the atoms are conglomerates of equally many hydrogen ions as their atom number was proposed already by William Prout in $1815^{24}$ but was abandoned until the discovery of isotopes gave it a new boost. Recently, number theory has given further strong support ${ }^{25}$, but ways and means of implementation have been hard to figure out. The present results would serve to suggest a solution consistent with known stages and events in the natural history of the elementary particles and in especial the electron-atom interrelations. The virtual extrusion mechanism can be empirically tested in terms of its output as will be done here in a periodic tabular form with special reference to the noble gases (Fig. 10).

Referring to Fig. 8a, the most marginal extrusion would be two singlets from one half of the terminal terrace, resulting in two hydrogen ions joining to a Hydrogen molecule (Fig. 10) which is then free to leave the stage. When at higher energy also two singlets have been forced together in the other half, the ensuing pulse would fuse to the Helium atom, likewise free to leave in any direction when there are no further singlets coming. When there are, the Helium motif would be deposited in the centre, and the remaining sending would line up peripheral to it. The sending is complete when half, i.e. eight, of the singlet positions in the next terrace (Fig. 6a) were filled and then transferred into the next thus saturated noble gas, i.e. Neon. And so it continues; Argon when the eight singlets in the remaining half of the terrace have been delivered; Krypton when the eighteen singlets in half of the next terrace 


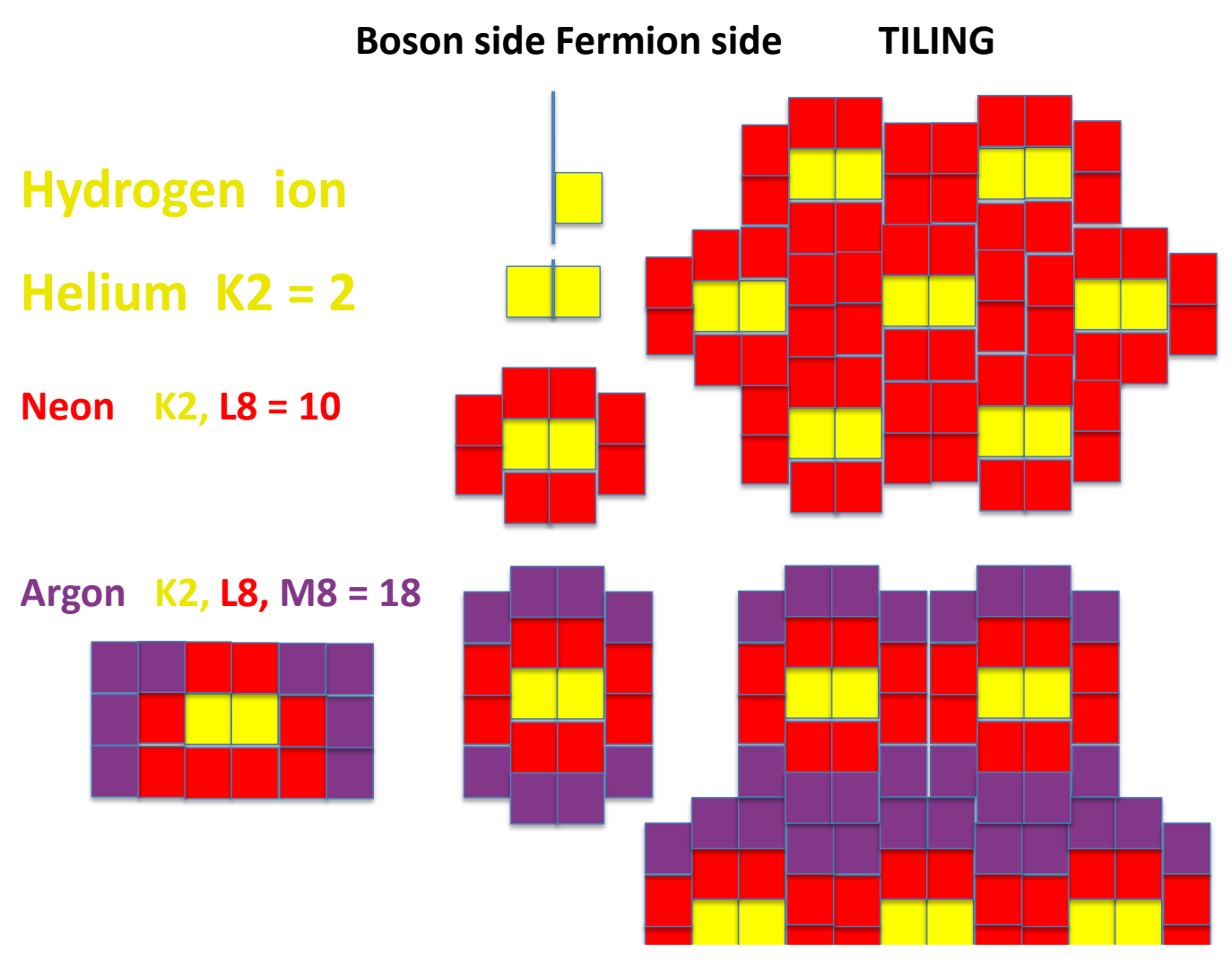

Krypton K2, L8, M18, N8 = $36 \quad$ Xenon K2, L8, M18, N18, O8 = 54

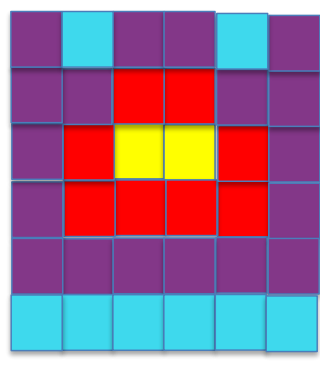

Radon K2, L8, M18, N32, O18, P8 = 86
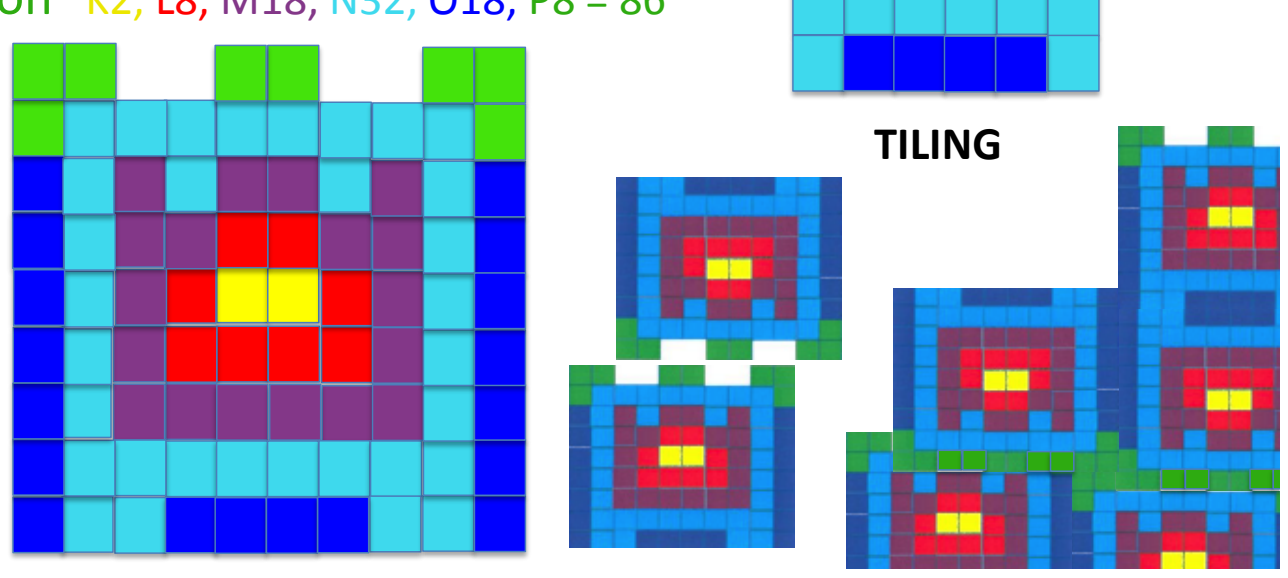


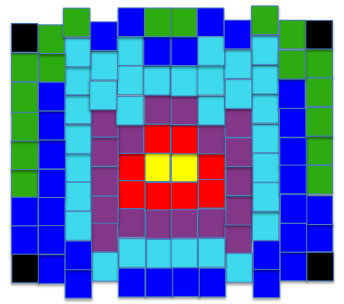

Ununoctium

K2, L8, M18, N32, O32, P18, Q8 = 118

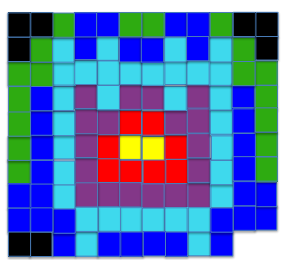

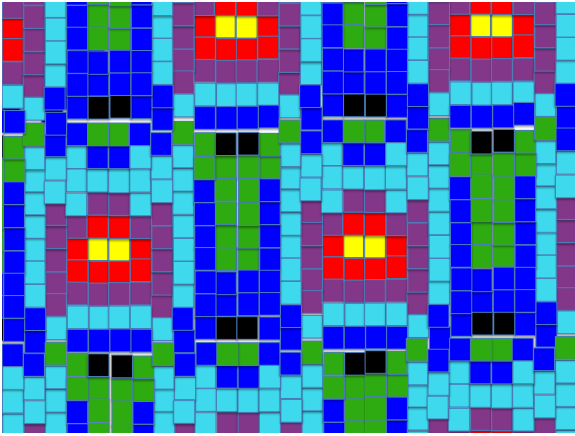

?-ium

K2, L8, M18, N32, O32, P18, Q10 = 120
TILING

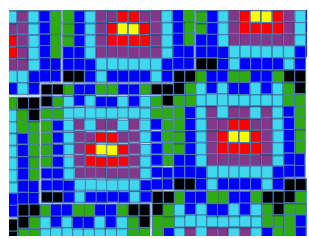

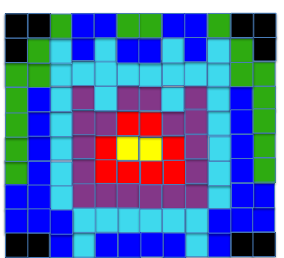

FIGURE 10. Periodic chart of the noble gases. Only one of the two initially transferred Hydrogen ions is shown to indicate its Fermion status. The coloring is according to the exaggerated color of the respective gas in the discharge tube. It is implied that the noble gases (like the other atoms) become saturated and released when so many primordial Hydrogen ions as their atom number have been pushed backwards in their self-similar global module expansion cone (Fig. 7a,b) till at last individually distributed in the corresponding singlet sites of the opening octahedron/tetrahedron mirror complex (Fig. 4a), from where they are virtually extruded as the origins for crystal nucleation of their motif in a separated order. A further condition of specifically the noble gases, discharged by their completion of the respective nucleogenetic cycle, is a spatial freedom and independence as expressed by direct tilability. Also other atoms along the margins in an ongoing cycle may be tilable as such but disposed to molecular coupling corresponding to the saturation status in the cycle.

have followed; Xenon when the eighteen in the remaining half have; and then there are 32 in half of the next terrace and when this number is added there is Radon. Then there should take 32 steps to the next state, but already after 28 occurs Flerovium - plausibly because at this high atom number it is still readily tiled (Fig. 10) - before at 32 the heaviest noble gas, Ununoctium really appears at atom number 118 .

Hence, the duplicated Bohr orbital model stratification of the periodic table is generated by the portioned delivery via a gradually narrowing condensation corridor from the virtual nozzle of which the pairwise dismantled Hydrogen ion seeds are deposited in a cross-sectional truss pattern and then expand within each so formed cell in a mode closely matching the "spiral...2D layer growth" 19,20 recently documented to operate at scales immediately bordering to the infinitesimal level and thus reasonably originating there. The scheme may seem far-fetched but is in effect quite akin to and not more complicated than the snowball packing by like inter-particle compression mechanism of repelling nucleons into a central nucleus, and instead of a reverse dispersal of bodiless electrons far out in the periphery integrates them in the process.

Only the noble gases are shown here, but the other atoms and their properties are possible to fit into their respective boxes in the present organically built, centrifugal periodic system, which once disclosed persists as a lasting alternative to the established tabular version. It doesn't share some of the deficiencies of this, e.g. having to have extra shelves for the Lanthanides and Actinides. These now line up in order along the margins of expanding cycles, and don't, except in positions like the halogens, differ much in chemical and other properties there; just as in reality. However, it is clear that the displayed charts are in a prototypal stage with many details still under elaboration, for instance, exact, including three-dimensional, accommodation of the marginal filling. But the 
principal and overall features make me, as a quite experienced and innovative Natural History researcher in quite pertaining related and overlapping fields (see http://www.ncbi.nlm.nih.gov/pubmed/?term=Trell+E) scientifically ascertained that future developments will be along this avenue, which also meets up with advancing Nanotechnology already able to witness in practice its ab initio pavement leading in just the present mold from the infinitesimal origin. Not only the periodic table, where much work, albeit partially under way, still remains, but in addition many properties of the atoms themselves are in the scope, like size, configuration and state - for example, solid in the highest number of cycles to atom completion, fluid in one cycle less and gaseous in two cycles less.

\section{DISCUSSION}

Nanotechnology is rapidly coming down in scale and has now reached the atomic level, ${ }^{19,20}$ all the way encountering hierarchically self-similar crystallographic lattice structures; usually, and in the inorganic realm almost exclusively of a regular solid composition identical to the present isotropic vector matrix generated via Marius Sophus Lie's original continuous geometriske transformationer. ${ }^{2,3}$ Thereby, Nanotechnology has carried to the very infinitesimal realm its rational Renaissance of un-dogmatic objective Natural History observation and operation whose deepest domain and idea can be summarized as extension: in the Aristotelian sense revived in Western science by Descartes and Lie alike. The latter even in his thesis stated that it "stands in an intimate dependence on philosophical reflections upon the nature of Cartesian Geometry",23 where, in turn, the first principle is that "the extension in length, width, and depth which constitutes the space occupied by a body, is exactly the same as that which constitutes the body...consequently, there cannot exist a space separate from body, since all spatial extension simply is body". ${ }^{26}$

The corollary is that space, which is extension, which is endless, consists of itself and is its own eigenelement and therefore necessarily space-filling in and by itself, and that its alternative is not emptiness or vacuum but nothing at all; the shrinking away and absence of any quality whatsoever, against which the direct straight line eigenvector of extension stands out as the categorical antithesis; the obligate obverse; equally immediate and raw and primary as its radical contrast. ${ }^{11}$ And Nanotechnology does not deal with vanishing points but with real extended objects thus rising as such from the outset, and Santilli brought extension to their mathematical formulations and formulas at birth ${ }^{4,5}$, and from there also opened up a de novo range of ground-up nanotechnological applications. ${ }^{6,27}$ My contribution is to show that one can indeed by solely a line and its maximally three-dimensional convolutions build a close corresponding facsimile with both ancient regular solid Cosmology and modern IVM Nanotechnology signature, bridging from the elementary particles to the elements and onwards.

\section{REFERENCES}

1. W. Killing, Matematische Annalen 34, 57-122 (1889).

2. M.S. Lie, "Over en classe geometriske transformationer," Ph.D. Thesis, Kristiania (now Oslo) University (1871).

3. E. Trell, Algebras Groups and Geometries 15, 395-445 (1998).

4. R.M. Santilli, Suppl. Nuevo Cimento 6, 1225-1249 (1968).

5. http://www.encyclopediaofmath.org/index.php/Lie-admissible_algebra

6. I. Gandzha and J. Kadisvily, New Science for A New Era (Sankata Printing Press, Kathmandu, 2011).

7. E. Trell, Acta Phys. Austriaca 55, 97-110 (1983).

8. E. Trell, Phys. Essays 4, 272-283 (1991).

9. E. Trell, Phys. Essays 5, 362-373 (1992).

10. E. Trell, Algebras, Groups and Geometries 15, 447-471 (1998).

11. E. Trell, AIP Conf. Proc. 1051, 127-141 (2008).

12. E. Trell, AIP Conf. Proc. 1479, 1037-1040 (2012).

13. E. Trell, J. Comp. Meth. Sci. Eng. 13, 245-270 (2013).

14. E. Trell, Bull. Calcutta Math. Soc. 97, 573-588 (2005).

15. E. Trell, AIP Conf. Proc. 1479, 2142-2146 (2012).

16. Grunch Net, Octet Truss, http//www.grunch.net/synergetics/octet.html

17. J. Werfel, K. Petersen and R. Nagpal, Science 343, 754-758 (2014). 
18. F. Martin, J. Fernandez, T. Havermeier, I. Foucar, Th. Weber et al., Science 315, 629-633 (2007).

19. P.D. Dandekar and M.F. Doherty, Science 344, 705-706 (2014).

20. A.I. Lupulescu and J.D. Rimer, Science 344, 729-732 (2014).

21. R.A al Aljouni, Development and evaluation of a digital tool for virtual reconstruction of historic Islamic geometric patterns. Dissertation, Texas A\&M University College Station (2005).

22. N. Bédaride and T. Fernique, arXiv:1208.3545v1, 1-7 (2012).

23. K.C. Cheung and N. Gershenfeld, Science 341, 1219-1221 (2013).

24. W. Prout, Ann. Philos. 6, 321-330 (1815).

25. J.C.A. Boeyens and D.C. Levendis, Number theory and the periodicity of matter, New York, Springer (2007).

26. Stanford Encyclopedia of Physics, Descarte's Physics http://plato.stanford.edu/entries/descartes-physics 27. E. Trell, Internat. J. Hydr. Energ. 28, 251-253 (2003). 